\title{
Merging Faces: A New Orthogonal Simplification of Solid Models
}

\author{
Irving Cruz-Matías and Dolors Ayala \\ Department de Llenguatges i Sistemes Informàtics, \\ Universitat Politècnica de Catalunya, Barcelona, Spain \\ \{icruz, dolorsa\}@lsi.upc.edu
}

\begin{abstract}
A new approach to simplify orthogonal pseudo-polyhedra (OPP) and binary volumes is presented. The method is incremental and produces a level-of-detail (LOD) sequence of OPP. Any object of this sequence contains the previous objects and, therefore, it is a bounding orthogonal approximation of them. The sequence finishes with the minimum axis-aligned bounding box (AABB). OPP are represented by the Extreme Vertices Model, a complete model that stores a subset of their vertices and performs fast Boolean operations. Simplification is achieved using a new approach called merging faces, which relies on the application of 2D Boolean operations. We also present a technique, based on the model continuity, for a better shape preservation. The method has been tested with several datasets and compared with two similar methods.
\end{abstract}

Keywords: Simplification, LOD, Bounding Volumes, Orthogonal Polyhedra, Binary volumes.

\section{Introduction}

The large size and complexity of the models often affects the computation speedup of their characteristics and their rendering efficiency. Simplification techniques can diminish these problems. Moreover, in some situations it is advantageous to exchange an exact geometric representation of an object for an approximated one, which can be processed more efficiently. Bounding structures are used for model simplification to accelerate tasks such as collision detection or distance computation. The most used bounding structures are AABB, spheres, oriented boxes or convex polyhedra. In this paper we present an approach to simplify OPP. The method computes a LOD sequence of bounding volumes (BV), that are also OPP, denoted as bounding OPP (BOPP). BOPP satisfy the two following properties: (1) any BOPP contains the previous one and (2) all the BOPP, as well as the original object, have the same AABB. Fig. 11depicts a 3D model and the obtained BOPP sequence, with the AABB for each one. We use the Extreme Vertices Model (EVM) to represent OPP. The presented simplification approach, called merging faces, relies on the application of 2D Boolean operations, which are fast using EVM, over the OPP faces. The presented method deals with general 3D orthogonal objects with any number of shells, cavities and through

R. Gonzalez-Diaz, M.-J. Jimenez, B. Medrano (Eds.): DGCI 2013, LNCS 7749, pp. 143-154, 2013.

(C) Springer-Verlag Berlin Heidelberg 2013 


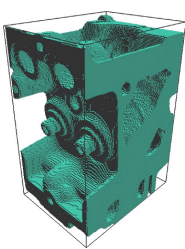

$92,922 \mathrm{EV}$

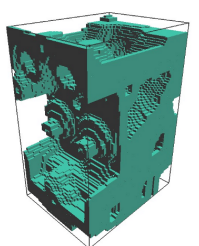

$18,346 \mathrm{EV}$

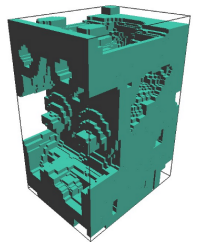

$8,768 \mathrm{EV}$

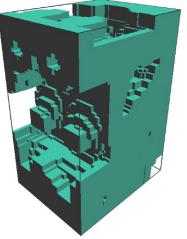

$3,948 \mathrm{EV}$

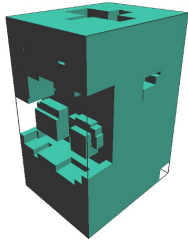

$802 \mathrm{EV}$

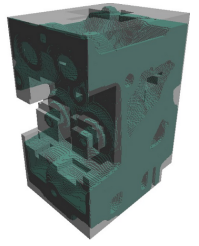

Fig. 1. A level-of-detail sequence of orthogonal pseudo-polyhedra (OPP) generated by our approach. From left to right: Original model, OPP with 19.7\%, 9.4\%, $4.2 \%$ and $0.8 \%$ of extreme vertices $(\mathrm{EV})$. In the last one, the 1st and 5 th objects are put together.

holes. We also develop a technique for a better shape preservation that avoids abrupt changes. The method has been tested with several datasets and compared with similar methods, showing satisfactory results.

\section{Related Work}

Model simplification has been extensively applied to triangular meshes [5] and extended to tetrahedral meshes evaluating the approximation error and the quality of the obtained mesh [4. Methods for LOD sequences of triangular and tetrahedral meshes can also be found extensively in the literature [16] as well as methods to simplify quadrilateral meshes 1121 . In contrast to these methods, that rely on geometric operations as edge-collapse or clustering, simplification can follow other strategies. Morphological operators as filleting and rounding can be used to simplify $2 \mathrm{D}$ binary images as well as $3 \mathrm{D}$ triangular meshes [26]. A carving strategy is applied to an octree model [23. as well as to a tetrahedral mesh [12] to simplify the topology. Simplification strategies have also been developed for B-Rep models [19] by removing connected sets of faces.

Several applications as collision detection [14, ray tracing 25] and volume of interest computation [9] use approximated shapes that are BV. Simple spheres [10] and AABB [20] are used as well as more sophisticated shapes as convex [15] or oriented 6] polytopes. Orthogonal polyhedra have also been proposed as BV [8] and as geometric bounds for CSG [1]. Orthogonally convex polygons are computed as orthogonal hulls for 2D images [3]. An orthogonal polygon is orthogonally convex if any axis-parallel line intersects it in at most one line segment. This problem has been extended to orthogonally convex polyhedra [2]

A sequence of BV can be obtained using alternative representations as octrees [18 23] resulting in a simplified geometry and topology, or BSP [13] obtaining a LOD sequence with a decreasing number of nodes. OP simplification has been carried out with a moving faces strategy that performs face displacements (but fails for objects with holes or more than one connected component) and the rectangle pairs strategy based on a box partition of the OP [8]. 


\section{Extreme Vertices Model (EVM)}

OP are two-manifold polyhedra with all their faces oriented in the three main axes. OPP are regular OP with a possible non-manifold boundary. General OPP are OPP with vertex coordinates having any value in $\mathbb{R}^{3}$. Polycubes are a subset of OPP all of whose vertices have integer coordinates, formed by joining one or more equal cubes (voxels) face to face. A 3D binary digital image represents an object as the union of its foreground voxels and its continuous analog is an OPP. In this paper we work with all these kind of objects represented with EVM. Let $Q$ be a finite set of points in $\mathbb{R}^{3}$, the $A B C$-sorted set of $Q$ is the set resulting from sorting $Q$ according to $A$-coordinate, then to $B$-coordinate, and then to $C$-coordinate. Let $P$ be an OPP, a brink is the maximal uninterrupted segment built out of a sequence of collinear and contiguous two-manifold edges of $P$ and its ending vertices are called extreme vertices (EV). An OPP can be represented in a concise way with the $A B C$-sorted set of its $\mathrm{EV}$ and such representation scheme is called EVM. EVM is a complete solid model [24.

Let $P$ be an OPP and $\Pi_{c}$ a plane whose normal is parallel, without loss of generality, to the $\mathrm{X}$ axis, intersecting it at $x=c$, where $c$ ranges from $-\infty$ to $\infty$. Then, this plane sweeps the whole space as $c$ varies within its range, intersecting $P$ at some intervals. Let us assume that this intersection changes at $c=c_{1}, \ldots, c_{n}$. More formally, $P \cap \Pi_{c_{i}-\delta} \neq P \cap \Pi_{c_{i}+\delta}, i=1, \ldots, n$, where $\delta$ is an arbitrarily small quantity. Then, $C_{i}(P)=P \cap \Pi_{c_{i}}$ is called a cut of $P$ and $S_{i}(P)=P \cap \Pi_{c_{s}}, c_{i}<c_{s}<c_{i+1}$, is called a section of $P$. Two cuts bounding a section $C_{i}$ and $C_{i+1}$ are called consecutive cuts. See Fig. 2. Sections can be computed from cuts and vice versa:

$$
\begin{array}{r}
\overline{S_{0}(P)}=\overline{S_{n}(P)}=\emptyset, \quad \overline{S_{i}(P)}=\overline{S_{i-1}(P)} \otimes^{*} \overline{C_{i}(P)}, i=1 \ldots n-1 \\
\overline{C_{i}(P)}=\overline{S_{i-1}(P)} \otimes^{*} \overline{S_{i}(P)}, i=1 \ldots n
\end{array}
$$

where $n$ is the number of cuts and $\otimes$ denotes the xor operation. Overline symbolizes the project operator, that projects a $d$-dimensional set of vertices lying on an orthogonal plane, like a cut or a section, onto the corresponding main plain, discarding their $d$ th coordinate. The star exponent ${ }^{*}$ denotes a regularized Boolean operation. A regularized set is defined as the closure of its interior. Regularized Boolean operations are needed to ensure 3D homogeneity [22].

Eq. 2 can be rewritten by expressing the $\otimes^{*}$ operation as the union of differences: $\overline{C_{i}(P)}=\left(\overline{S_{i-1}(P)}-{ }^{*} \overline{S_{i}(P)}\right) \cup^{*}\left(\overline{S_{i}(P)}-{ }^{*} \overline{S_{i-1}(P)}\right)$, and any cut can be decomposed into its forward difference $(F D)$ and backward difference $(B D)$ :

$$
\overline{F D_{i}(P)}=\overline{S_{i-1}(P)}-{ }^{*} \overline{S_{i}(P)}, \quad \overline{B D_{i}(P)}=\overline{S_{i}(P)}-{ }^{*} \overline{S_{i-1}(P)}, i=1 \ldots n
$$

$F D_{i}(P)$ is the set of $C_{i}(P)$ faces whose normal vector points to the positive side of the coordinate axis perpendicular to $C_{i}(P)$ and $B D_{i}(P)$ is the set of faces whose normal vector points to the negative side (see Fig. 22). This property guarantees the correct orientation of faces and the computation of the non-EV. EVM Boolean operations are computed by applying recursively (in $n \mathrm{D}$ ) the same Boolean operation over the $(n-1) \mathrm{D}$ OPP sections. The base case performs this 

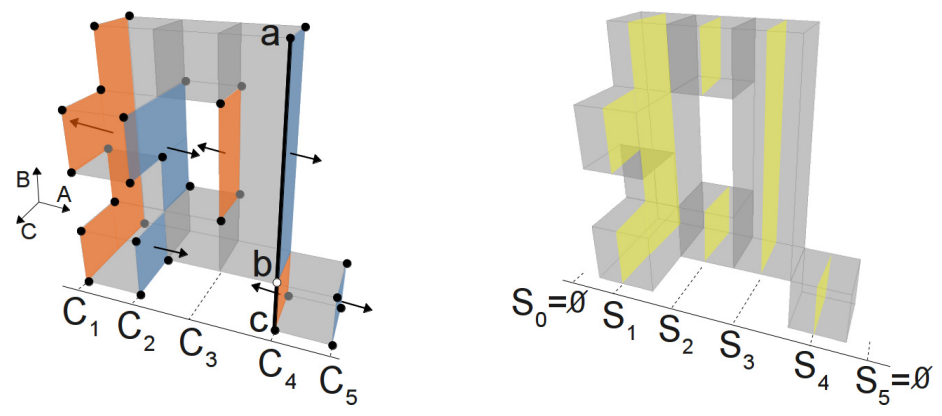

Fig. 2. An EVM-encoded ( $A B C$-sorted) OPP. Left: EV marked with dots, cuts decomposed in FD (blue) and BD (orange), normal vectors represented with arrows; a vertical brink from vertex $\mathbf{a}$ to $\mathbf{c}$ is marked showing that these vertices are both EV while the vertex $\mathbf{b}$ is a non-EV. Right: The sections of the object highlighted in yellow.

operation in 1D. The $\otimes^{*}$ is even faster, $E V M\left(P \otimes^{*} Q\right)=E V M(P) \otimes^{*} E V M(Q)$, i.e, it is a simple point-wise xor, without section computation. For more details concerning EVM see [1] and [17.

\section{Algorithm Overview}

Let $B(P)$ be the bounding orthogonal pseudo-polyhedron (BOPP) of an OPP $P$ and let $\phi_{0}$ be an initial OPP. A finite sequence $\phi_{1}, \phi_{2}, \ldots, \phi_{p}$ of OPP is generated that fulfill the following properties:

1. $\phi_{i+1}=B\left(\phi_{i}\right), i=0 \ldots p-1$

2. $\phi_{i} \subseteq B\left(\phi_{i}\right), i=0 \ldots p-1$, and, therefore, $\phi_{i} \subseteq \phi_{i+1}, i=0 \ldots p-1$

3. $\phi_{p}=A A B B\left(\phi_{i}\right), i=0 \ldots p$

The first property indicates that the approach is incremental. The second one, called subset property, is intrinsic in bounding structures. The last property states that the sequence is finite and that ends with the AABB that is shared by all the OPP of the sequence.

The simplification strategy, merging faces, works with pairs of consecutive cuts of $P$. For each cut of a pair, a displacement to its faces in the direction of their corresponding normal vector is applied and, then, the displaced faces are merged with those faces of the other cut with the same normal vector. The process is controlled by the displacement parameter, $d$, that indicates the maximum displacement allowed, in such a way that only pairs of consecutive cuts that are at a distance $\leq d$ are actually merged. If $P$ is represented as an $A B C$-sorted EVM, the application of this process only will coarsen $P$ in the $A$-coordinate. Therefore to obtain $B(P)$ the process is repeated for the other two main directions. The result can be slightly different depending on the ordering in which the three main directions are selected. In order to speed up the computation, the best first candidate would be the $A B C$-ordering with $A$-axis having less number of 
cuts, but as EVM does not report this value, it can be approximated by $c_{n}-c_{1}$, $c_{1}$ and $c_{n}$ being respectively the coordinates of the first and last cut in this direction. We can compute the whole sequence of BOPP or ask for a BOPP with a maximum number of EV.

The input of the whole method is an EVM represented OPP, $P$, which corresponds to the initial object $\phi_{0}$ and the desired maximum number of $\mathrm{EV}, n v$, in the simplified model, and returns an EVM represented OPP, $Q$, corresponding to the object $\phi_{k}$ of the sequence, which has no more than $n v \mathrm{EV}$. As the method is incremental, it actually computes all the objects between $\phi_{1}$ and $\phi_{k}$. It performs $k$ times the merging faces process for successive values of $d, d=d_{i}, i=1, \ldots, k$, obtaining the corresponding $\phi_{i}$. Displacements $d_{i}$ can be in any units and incremented in any quantity. For OPP corresponding to digital images the basic unit is one voxel, i.e. $d_{i}=i$. For general OPP, with float coordinate values, $d_{i}$ can take any values ranging from the minimum distance between cuts and the AABB size. Note that if the increment of $d$ is too large, far apart consecutive cuts can be merged in early iterations causing abrupt changes in the simplified object. The next pseudocode shows the iterative algorithm and concerning $d$ considers digital images. Get_nev() returns the number of EV of the given object $P$, mergingFaces() receives the object $P$ and a distance $d$, and returns the object $Q=B(P)$, merging pairs of consecutive cuts at a distance $\leq d$. To compute the whole LOD sequence of BOPP, $n v$ must be 8 .

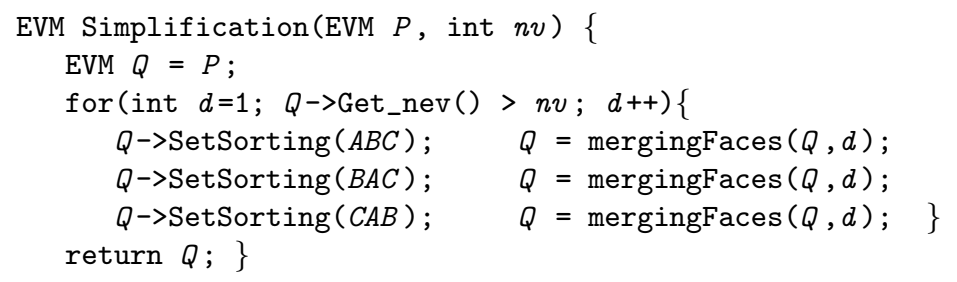

\section{$5 \quad$ Merging Faces Approach}

In this section we first explain the basic process, then the treatment of the void space and finally, we discuss the way to select pairs of cuts. Let $P$ be an OPP and let $C_{A}$ and $C_{B}$ be two consecutive cuts of $P$ with $F D_{A}, B D_{A}, F D_{B}, B D_{B}$ as their corresponding forward and backward differences (see Eq. 3). To obtain a coarsened OPP, the merging faces process displaces $B D_{B}$ to the position of $B D_{A}$, and $F D_{A}$ to the position of $F D_{B}$. Then the new cuts $n e w C_{A}$ and new $C_{B}$ that will replace $C_{A}$ and $C_{B}$, respectively, in the input model, are computed as:

$$
\overline{n e w C_{A}}=\overline{B D_{A}} \cup^{*} \overline{B D_{B}}, \quad \overline{n e w C_{B}}=\overline{F D_{A}} \cup^{*} \overline{F D_{B}}
$$

This process fulfills the properties stated in Sec. 4. Face displacements are done in the direction of their respective normal vector, i.e. outward of the object. Then, for any $P, P \subseteq B(P)$ (subset property). The property concerning AABB can be proved by considering that the AABB of an OPP can be defined as 


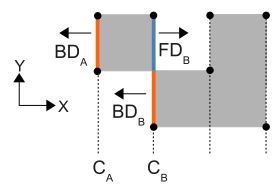

(a)

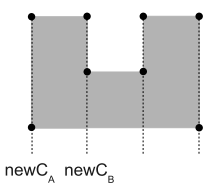

(b)

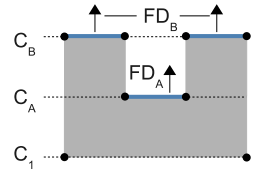

(c)

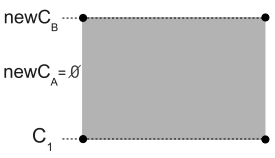

(d)

Fig. 3. 2D example: (a) Faces merged in a first step. (b) Result after applying Eq. 4 and end for XY-sorting. (c) Faces merged by YX-sorting. (d) Resulting BOPP.

the intersection of the six planes corresponding to the first and last cut in the three main directions, and the fact that the object will never extend beyond the first and last cut of the initial object because the displacements are bounded by these cuts. Moreover, in the last iteration, the first cut of $\phi_{p}$ (the AABB), will correspond to the union of all BD of all cuts, and likewise the last cut to the union of all FD. Fig. 3 shows a 2D example where the process is applied.

An OPP may have any number of rings on faces, through holes and shells. In these cases, Eq. 4 could not give the desirable result, for instance, the object in Fig. 4(a), remains the same after the application of Eq. 4 . To deal with this issue, we first detect and then close void spaces. Given $C_{A}$ and $C_{B}, B D_{A}$ and $B D_{B}$ are sets of faces whose normal vectors point to the opposite direction of $F D_{A}$ and $F D_{B}$, but $F D_{A}$ and $B D_{B}$ define a void space as $v \operatorname{Space}\left(C_{A}, C_{B}\right)=$ $\overline{F D_{A}} \cap * \overline{B D_{B}}$. Removing $v$ Space from both $n e w C_{A}$ and $n e w C_{B}$, closes the void space between $C_{A}$ and $C_{B}$. Then, Eq. 4 is extended as:

$$
\begin{aligned}
& \overline{\text { newC } C_{A}}=\left(\overline{B D_{A}} \cup^{*} \overline{B D_{B}}\right)-{ }^{*}\left(\overline{F D_{A}} \cap^{*} \overline{B D_{B}}\right) \\
& \overline{\text { newC } C_{B}}=\left(\overline{F D_{A}} \cup^{*} \overline{F D_{B}}\right)-{ }^{*}\left(\overline{F D_{A}} \cap^{*} \overline{B D_{B}}\right)
\end{aligned}
$$

Observe that when $v$ Space $=\emptyset$, Eq. 5 and 4 are equivalent. The subset and the AABB properties are also guaranteed as we remove interior void spaces. Fig. 4(a) shows an example with a single hole, here $\overline{F D_{A}}=\overline{B D_{B}}$ and $v \operatorname{Space}\left(C_{A}, C_{B}\right)=$ $\overline{F D_{A}}=\overline{B D_{B}}$. Then, applying Eq. 5. new $C_{A}=\emptyset$ and the hole is closed (Fig. 4(b)). In Eq. 5, basic merging and void space removal are performed jointly. Some void spaces, as the simple concavity in Fig. 4(c), are solved with Eq. 4 . However, general void spaces, as those in Fig. 4 (d-f), require the application of Eq. 5. Some void spaces can be detected in all three directions, as the cavity depicted in Fig. 4(f) but some others are only detected in one or two directions. Fig. 4( $(\mathrm{g}-\mathrm{j})$ show a working example in an object with through holes.

Two EVM properties, that state that for two special cases union and difference consist in simple point-wise xor operations [1], together with two EVM theorems, permit to rewrite Eq. 5 in a way faster to compute.

- Property 1: Let $P$ and $Q$ be two OPP such that $P \cap^{*} Q=\emptyset$, having $E V M(P)$ and $E V M(Q)$ as their models, then $E V M\left(P \cup^{*} Q\right)=E V M(P) \otimes^{*} E V M(Q)$.

- Property 2: Let $P$ and $Q$ be two OPP such that $P \supseteq Q$, with $E V M(P)$ and $\operatorname{EVM}(Q)$ as their models, then $E V M\left(P-{ }^{*} Q\right)=E V M(P) \otimes^{*} E V M(Q)$. 


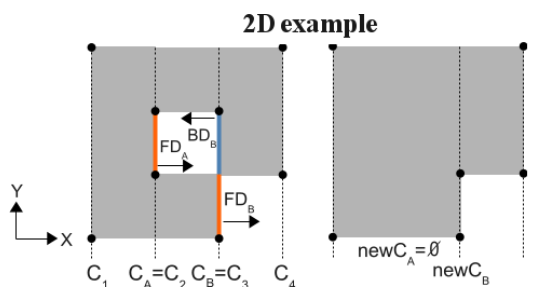

(a)Faces merged in order (b) Result and end to close the hole. for the XY-sorting.

Some configurations of void spaces in 3D

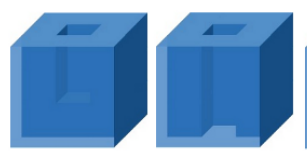

(c) Concavity

(d) Through hole

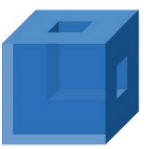

(e) Through hole in L-shape

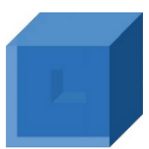

(f) Internal hole or cavity

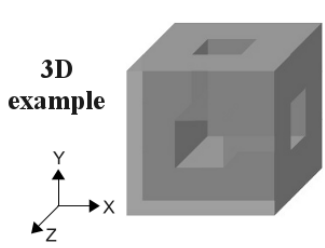

(g) Original object.

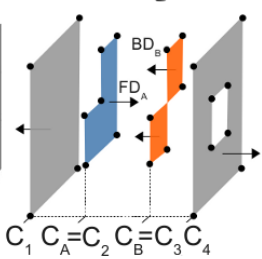

(h) Faces merged in a first step.

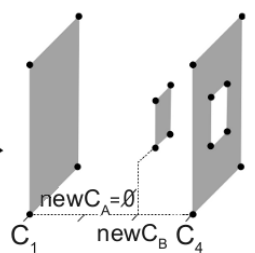

(i) Resulting cuts.

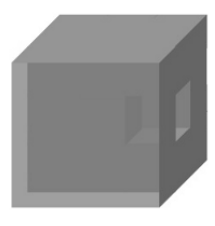

(j) Result for the XYZ-sorting.

Fig. 4. Merging faces and treatment of void space

Theorem 1. The projection of $F D$ and $B D$ of two consecutive cuts $C_{A}$ and $C_{B}$ are quasi-disjoint sets respectively, i.e. $\overline{F D_{A}} \cap * \overline{F D_{B}}=\emptyset$ and $\overline{B D_{A}} \cap * \overline{B D_{B}}=\emptyset$.

Proof. The proof is based on the Jordan theorem and the fact that any ray crossing the boundary of the polyhedron, alternatively goes from outside to inside and vice versa. Therefore, in any $\mathrm{OPP}$, assuming that $\overline{F D_{A}} \cap^{*} \overline{F D_{B}} \neq \emptyset$, would mean that a ray could cross $F D_{A}$ going outside and then cross $F D_{B}$ going outside again, which is a contradiction. The same reasoning applies to BD.

Theorem 2. vSpace $\left(C_{A}, C_{B}\right) \subseteq\left(\overline{B D_{A}} \cup * \overline{B D_{B}}\right)$

Proof. vSpace $\left(C_{A}, C_{B}\right)=\overline{F D_{A}} \cap * \overline{B D_{B}}$. Without loss of generality:

$\left(\overline{F D_{A}} \cap^{*} \overline{B D_{B}}\right) \subseteq \overline{B D_{B}} \subseteq\left(\overline{B D_{A}} \cup * \overline{B D_{B}}\right)$, and thus:

$\left(\overline{F D_{A}} \cap^{*} \overline{B D_{B}}\right) \subseteq\left(\overline{B D_{A}} \cup * \overline{B D_{B}}\right)$

In a similar way $v \operatorname{Space}\left(C_{A}, C_{B}\right) \subseteq\left(\overline{F D_{A}} \cup^{*} \overline{F D_{B}}\right)$ can be proved.

According to these theorems and the EVM properties, Eq. 5 is rewritten as:

$$
\begin{aligned}
& \overline{\text { newC } C_{A}}=\overline{B D_{A}} \otimes^{*} \overline{B D_{B}} \otimes^{*}\left(\overline{F D_{A}} \cap^{*} \overline{B D_{B}}\right) \\
& \overline{n e w C_{B}}=\overline{F D_{A}} \otimes^{*} \overline{F D_{B}} \otimes^{*}\left(\overline{F D_{A}} \cap^{*} \overline{B D_{B}}\right)
\end{aligned}
$$

Merging faces takes pairs of consecutive cuts but we must establish the way in which they are selected. After analyzing several alternatives, we selected the one with best visual results. It consists in taking cuts two by two: $\left(C_{A}=C_{i}, C_{B}=\right.$ $\left.C_{i+1}\right), i=1, i \leq n-1, i=i+2$, i.e. first the pair $\left(C_{1}, C_{2}\right)$, then $\left(C_{3}, C_{4}\right)$, and so on. However, when $C_{A}$ and $C_{B}$ are such that $F D_{A}=B D_{B}=\emptyset$, merging faces has no effect (e.g. consider $C_{A}=C_{1}$ and $C_{B}=C_{2}$ in Fig. $4(\mathrm{~h})$ ). On the other hand, 
only pairs of cuts at a distance $\leq d$ are processed. Then, given the pair of cuts $\left(C_{A}=C_{i}, C_{B}=C_{i+1}\right)$, if Exp. 7 is fulfilled, this pair is processed and the method continues with the pair $\left(C_{i+2}, C_{i+3}\right)$. Otherwise $C_{i}$ is copied to the resulting OPP and the method continues with the pair $\left(C_{i+1}, C_{i+2}\right)$. Moreover, for symmetry preservation purposes, the model is analyzed from both sides at a time.

$$
\left(F D_{A} \neq \emptyset \text { or } B D_{B} \neq \emptyset\right) \text { and distance }\left(C_{A}, C_{B}\right) \leq d
$$

\section{Shape Preservation}

Shape preservation is an important aspect in model simplification. Merging two cuts is performed in all their extension, but there are parts of these cuts that are isolated and merging them could result in too abrupt changes. Let $\left(C_{A}, C_{B}\right)$ be the pair of cuts to be merged, we will refer as isolated faces those faces of $C_{A}$ whose projections do not share either an edge or a vertex with others in $C_{B}$ and vice versa. Removing these faces of the merging process results in a better approximation (see Fig. [5 (d-f)). Let $I_{A}$ and $I_{B}$ be the isolated faces of $C_{A}$ and $C_{B}$ respectively, the new $F D_{A}^{\prime}, B D_{A}^{\prime}, F D_{B}^{\prime}$ and $B D_{B}^{\prime}$ are computed according to Eq. 8. These values are used in Eq. 6] to generate new $C_{A}^{\prime}$ and $n e w C_{B}^{\prime}$, and after that, the isolated faces can be reintegrated with a union operation. However, by definition $I_{A}$ and $C_{A}$ are disjoint sets and therefore, $I_{A}$ and new $C_{A}^{\prime}$ are also disjoint sets (the same applies to $I_{B}, C_{B}$ and $n e w C_{B}^{\prime}$ ). Then, according to Property 1, an xor operation is performed instead (See Eq. 9).

$$
\begin{aligned}
\overline{F D_{A}^{\prime}} & =\overline{F D_{A}}-{ }^{*} \overline{I_{A}}, & & \overline{B D_{A}^{\prime}}=\overline{B D_{A}}-{ }^{*} \overline{I_{A}} \\
\overline{F D_{B}^{\prime}} & =\overline{F D_{B}}-* \overline{I_{B}}, & & \overline{B D_{B}^{\prime}}=\overline{B D_{B}}-{ }^{*} \overline{I_{B}} \\
\overline{n e w C_{A}} & =\overline{n e w C_{A}^{\prime}} \otimes * \overline{I_{A}}, & & \overline{n e w C_{B}}=\overline{n e w C_{B}^{\prime}} \otimes * \overline{I_{B}}
\end{aligned}
$$

Fig. $5(\mathrm{a}-\mathrm{c})$ show an example where an isolated face $(i f)$ is depicted. Note that if remains in place, and only those faces that share either an edge or a vertex are taken into account throughout the merging faces process. The application of this technique does not affect the subset property (see Sec. 41). However, the property concerning finiteness cannot be guaranteed, as there may be consecutive

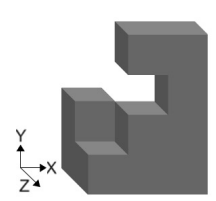

(a)

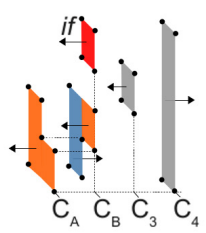

(b)

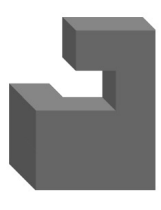

(c)

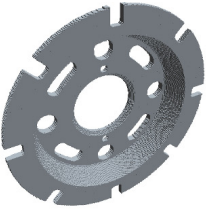

(d) $55,246 \mathrm{EV}$

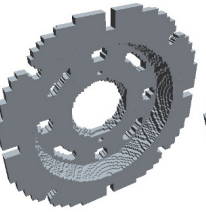

(e) $7,578 \mathrm{EV}$

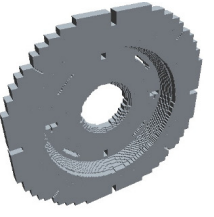

(f) $7,772 \mathrm{EV}$

Fig. 5. Shape preservation technique. (a) A simple 3D model. (b) All cuts and EV; in red an isolated face. (c) Result of applying merging faces with the technique to the pair $\left(C_{A}, C_{B}\right)$. (d) Original DiskBrake model. (e) and (f) BOPP with and without the shape preservation technique respectively, both having less than $15 \%$ of $\mathrm{EV}$. 
cuts where all the faces are isolated and, in this case, we would not reach the AABB. Therefore, when all the faces are isolated, we do not apply the shape preservation technique and use directly Eq. 6. To detect isolated faces between $C_{A}$ and $C_{B}$, we check each face of $C_{A}$ against each face of $C_{B}$. For this process we use a decomposition model, the Compact Union of Disjoint Boxes (CUDB) 7], which is derived from EVM by splitting the orthogonal faces into a set of ABsorted rectangles. We have implemented an algorithm for rectangle adjacency detection. As a 2D CUDB is a sorted set of rectangles, we apply a merging process, i.e., we perform a traversal of the CUDB models of each face in $C_{A}$ and $C_{B}$ at a time and check for rectangle intersection. Thus, if we have $n$ faces with $m_{i}$ boxes each one, the worst case time-complexity is $O(n \cdot M), M=\sum_{i=1}^{n} m_{i}$.

\section{$7 \quad$ Results and Discussion}

We have tested merging faces with and without shape preservation in several $3 \mathrm{D}$ datasets with different shape features, obtained from public repositories and our own collection. All of them have been converted to EVM using existing algorithms [17. The presented algorithms have been written in $\mathrm{C}++$ and executed on a PC Intel@Core i7 CPU 870 at $2.93 \mathrm{GHz}$ with $16 \mathrm{~Gb}$ of RAM under Linux. The computation time of merging faces depends directly on the number of EV which is related to how well aligned is the object with respect to the three axes. This fact is shared by most of the EVM-based developed methods.

Table1 1 shows the obtained results. Note that merging faces with shape preservation requires, in general, more time than without it. However, in some cases the time is almost the same (DiskBrake) or even less (Pegasus, Dragon). This is due to that in some datasets the application of shape preservation results in a significant reduction of the number of processed pairs and, consequently, in the execution time. Figures 1 and 6 depict some of these datasets with several BOPP of their LOD sequence using shape preservation. We consider EV as the basic geometric element and these figures show the number of EV and the percentage of reduction with respect to the number of EV the original dataset. We can observe that BOPPs with less than $25 \%$ of EV give a very good approximation of

Table 1. For each dataset: size in voxels and number of EV $(|E V|)$; maximum distance $d$, number of processed pairs $n p$ and time $T$ to compute the whole LOD sequence without shape preservation; $d_{s p}, n p_{s p}$ and $T_{s p}$ : same values with shape preservation

\begin{tabular}{|l|r|r||r|r|r||r|r|r|}
\hline Dataset & Size & $|E V|$ & $d$ & $n p$ & $T($ sec. $)$ & $d_{s p}$ & $n p_{s p}$ & $T_{s p}($ sec. $)$ \\
\hline DoorPieces & $267 \times 394 \times 72$ & 11,784 & 46 & 894 & $\mathbf{0 . 3 5}$ & 46 & 953 & 0.47 \\
\hline Bunny & $127 \times 128 \times 98$ & 24,796 & 24 & 1,098 & $\mathbf{0 . 6 3}$ & 30 & 1,236 & 0.85 \\
\hline Foot & $96 \times 270 \times 97$ & 39,078 & 55 & 2,031 & $\mathbf{1 . 3 2}$ & 49 & 2,009 & 1.73 \\
\hline DiskBrake & $299 \times 300 \times 43$ & 55,246 & 32 & 2,335 & $\mathbf{1 . 8 5}$ & 35 & 2,173 & 1.95 \\
\hline Engine & $140 \times 197 \times 108$ & 92,922 & 19 & 2,189 & $\mathbf{3 . 0 8}$ & 22 & 2,761 & 4.95 \\
\hline Pegasus & $382 \times 512 \times 367$ & 290,916 & 103 & 20,782 & 37.85 & 137 & 9,228 & $\mathbf{1 9 . 5 5}$ \\
\hline Dragon & $511 \times 360 \times 228$ & 314,290 & 56 & 10,646 & 19.01 & 57 & 8,325 & $\mathbf{1 8 . 4 2}$ \\
\hline
\end{tabular}




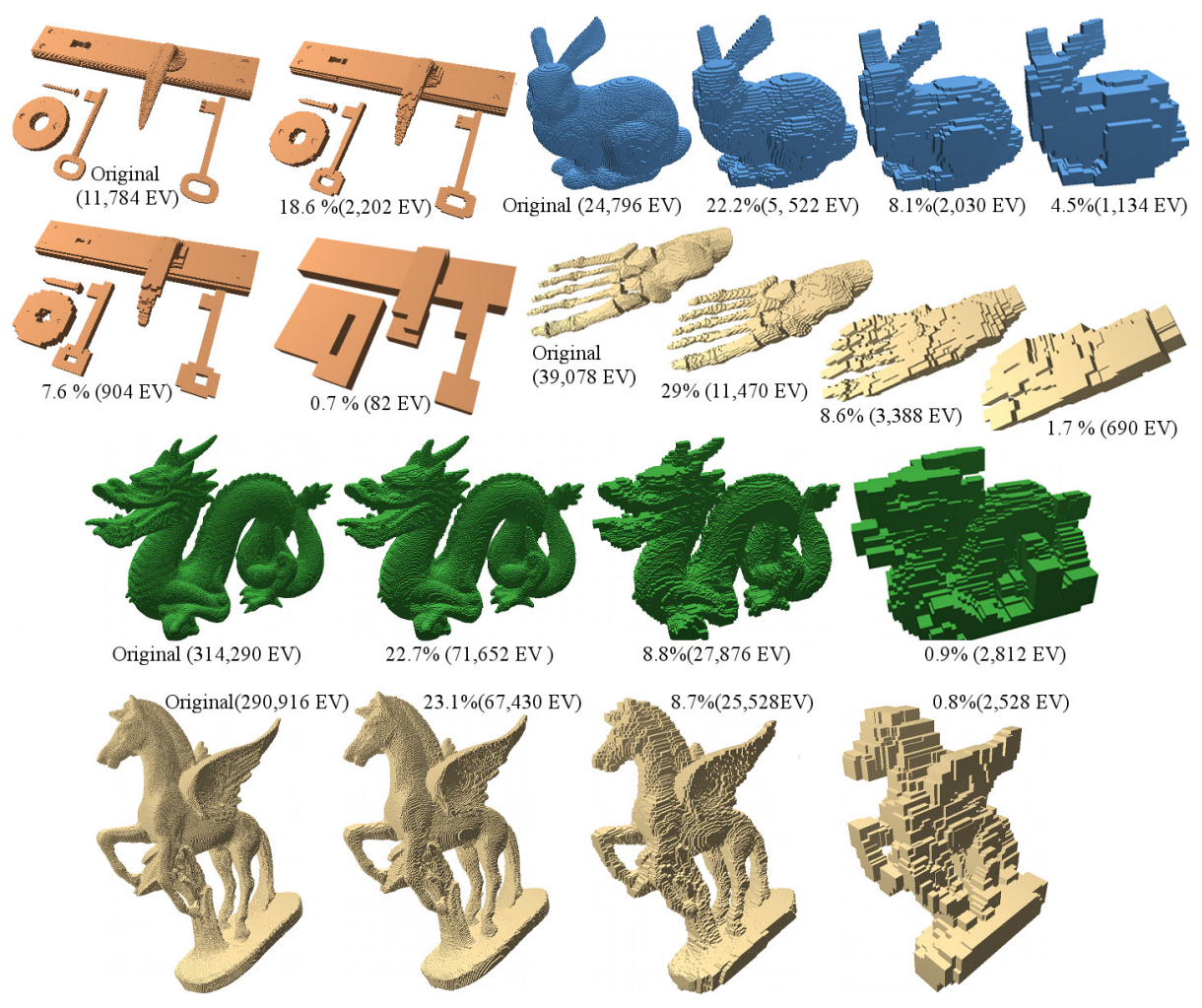

Fig. 6. Some results of the tested models, showing the number of extreme vertices

the objects, that BOPPs with less than $10 \%$ yield an acceptable approximation, and, although BOPPs with less than $1 \%$ look blocky, they still give an indication of the object shape. Also note how the holes progressively get closed, specially in Engine and DoorPieces and the connected components are joined in DoorPieces.

We have compared the presented method against two similar methods that compute bounding volumes (see Figs. 6 and 7). We compare the experimental results in terms of percentage of basic geometric elements reduction versus visual approximation. The first method (OCT) $[18$ is an octree-based approximation and compression method for 3D objects. Approximations are obtained by truncating the octree. The results of the method are presented in terms of number of blocks (octree nodes), which is the basic geometric element of the octree and the test model used is the Bunny dataset. The original model requires 29,007 blocks and 24,796 EV. Figures 7 (left) show two objects generated by OCT. We can see that merging faces gives a better indication of the shape with less than $5.1 \%$ of elements than OCT, and even an object with $22.2 \%$ gives a better approximation than OCT with $52 \%$. The second method (BSP) 13 is a progressive solid simplification of objects represented by a Binary Space Partition tree. It uses a volume bounded convex simplification and a plane collapse method to reduce 

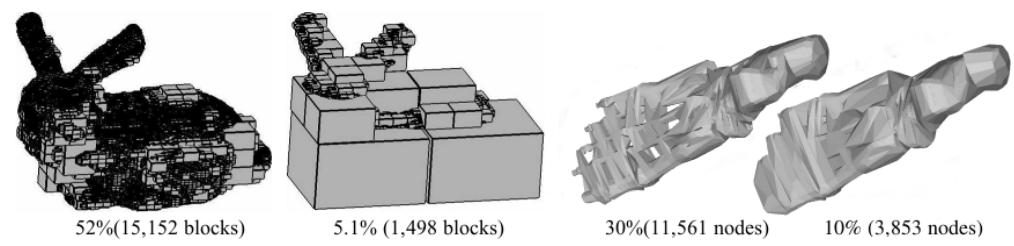

Fig. 7. Some results of OCT (Bunny dataset) and BSP (Foot dataset) methods, showing the number of elements, blocks and BSP nodes respectively. Figures have been taken from the references [18]13].

the BSP-tree depth. The basic geometric element is a plane (BSP node). We have used the most of the datasets presented in this work and converted them to EVM in such a way that the number of EV of the original model is appoximately the same than the number of planes in the BSP tree. We report here the results of the Foot dataset. The original model has 39,078 EV and 38,535 planes. Figures 7 (right) show two LOD-objects generated by BSP. We can observe that the shape of the fingers in merging faces is preserved until the $1.7 \%$ version while in the BSP method the fingers look wrapped since the $30 \%$ version. Similar results have been obtained with the other datasets.

\section{Conclusions}

We have proposed an approach to simplify orthogonal polyhedra and binary images, represented with the EVM. It generates a LOD sequence of BOPP, fulfilling the common subset and AABB properties of bounding structures. The approach is based on a merging strategy that involves pairs of consecutive cuts. We have showed that our approach can deal with objects with any number of holes and connected components, and presented a technique, based on the model continuity, for a better shape preservation. We have compared our method against two similar methods, and in general, our method gives better approximations with the same number of basic geometric elements. Directions for future work include the study of a lossless simplification approach based on the presented one.

Acknowledgements. This work was partially supported by the national projects TIN2008-02903 and TIN2011-24220 and a MAEC-AECID grant for I. Cruz-Matías, all of the Spanish government. The authors thank the anonymous reviewers whose remarks and suggestions have allowed to greatly improve the paper.

\section{References}

1. Aguilera, A., Ayala, D.: Orthogonal Polyhedra as Geometric Bounds in CSG. In: IVth Symp. on Solid Modeling and Appl., pp. 56-67. ACM (1997)

2. Biedl, T., Genç, B.: Reconstructing orthogonal polyhedra from putative vertex sets. Computational Geometry 44(8), 409-417 (2011) 
3. Biswas, A., et al.: A linear-time combinatorial algorithm to find the orthogonal hull of an object on the digital plane. Information Sciences 216, 176-195 (2012)

4. Cignoni, P., et al.: Simplification of tetrahedral meshes with accurate error evaluation. In: Proceedings of the IEEE Visualization Conference, pp. 85-92 (2000)

5. Cignoni, P., Montani, C., Scopigno, R.: A comparison of mesh simplification algorithms. Computers and Graphics 22(1), 37-54 (1998)

6. Coming, D.S., Staadt, O.G.: Velocity-aligned discrete oriented polytopes for dynamic collision detection. IEEE Trans. Vis. and Computer Graphics 14, 1-12 (2008)

7. Cruz-Matías, I., Ayala, D.: CUDB: An improved decomposition model for orthogonal pseudo-polyhedra. Tech. Rep. LSI-11-2-T, UPC (2011)

8. Esperança, C., Samet, H.: Orthogonal Polygons as Bounding Structures in FilterRefine Query Processing Strategies. In: Scholl, M.O., Voisard, A. (eds.) SSD 1997. LNCS, vol. 1262, pp. 197-220. Springer, Heidelberg (1997)

9. Fuchs, R., Welker, V., Hornegger, J.: Non-convex polyhedral volume of interest selection. J. of Computerized Medical Imaging and Graphics 34(2), 105-113 (2010)

10. Gagvani, N., Silver, D.: Shape-based volumetric collision detection. In: VVS 2000: Proceedings of the 2000 IEEE Symp. on Volume Visualization, pp. 57-61. ACM Press (2000)

11. Greß, A., Klein, R.: Efficient representation and extraction of 2-manifold isosurfaces using kd-trees. Graphical Models 66, 370-397 (2004)

12. Hagbi, N., El-Sana, J.: Carving for topology simplification of polygonal meshes. Comput. Aided Des. 42, 67-75 (2010)

13. Huang, P., Wang, C.: Volume and complexity bounded simplification of solid model represented by binary space partition. In: Proc. SPM 2010, pp. 177-182 (2010)

14. Jiménez, P., Thomas, F., Torras, C.: 3D collision detection: A survey. Computers and Graphics 25(2), 269-285 (2000)

15. Klosowski, J.T., et al.: Efficient collision detection using bounding volume hierarchies of k-dops. IEEE Trans. Vis. and Computer Graphics 4(1), 21-36 (1998)

16. Ripolles, O., Chover, M., Gumbau, J., Ramos, F., Puig, A.: Rendering continuous level-of-detail meshes by masking strips. Graphical Models 71(5), 184-195 (2009)

17. Rodríguez, J., Ayala, D., Aguilera, A.: EVM: A Complete Solid Model for Surface Rendering. In: Geometric Modeling for Scientific Vis., pp. 259-274. Springer (2004)

18. Samet, H., Kochut, A.: Octree approximation and compression methods. In: Proceedings of the 1st Int. Symp. 3DPVT, pp. 460-469. IEEE Computer Society (2002)

19. Sun, R., Gao, S., Zhao, W.: An approach to b-rep model simplification based on region suppression. Computers \& Graphics 34(5), 556-564 (2010)

20. Suri, S., Hubbard, P.M., Hughes, J.F.: Analyzing bounding boxes for object intersection. ACM Trans. Graph. 18, 257-277 (1999)

21. Tarini, M., Pietroni, N., Cignoni, P., Panozzo, D., Puppo, E.: Practical quad mesh simplification. Computer Graphics Forum 29(2), 407-418 (2010)

22. Tilove, R., Requicha, A.: Closure of boolean operations on geometric entities. Computer-Aided Design 12(5), 219-220 (1980)

23. Vanderhyde, J., Szymczak, A.: Topological simplification of isosurfaces in volume data using octrees. Graphical Models 70, 16-31 (2008)

24. Vigo, M., Pla, N., Ayala, D., Martínez, J.: Efficient algorithms for boundary extraction of 2D and 3D orthogonal pseudomanifolds. Grap. Models 74, 61-74 (2012)

25. Wald, I., Boulos, S., Shirley, P.: Ray tracing deformable scenes using dynamic bounding volume hierarchies. ACM Trans. Graph. 26 (January 2007)

26. Williams, J., Rossignac, J.: Tightening: Morphological simplification. International Journal of Computational Geometry \& Applications 17(5), 487-503 (2007) 